\title{
Probing a bino NLSP at lepton colliders
}

\author{
Junmou Chen, ${ }^{1, \dagger}$ Chengcheng Han, ${ }^{2, \dagger}$ Jin Min Yang ${ }^{3,4}$ and Mengchao Zhang $\oplus^{1, *}$ \\ ${ }^{1}$ Department of Physics and Siyuan Laboratory, Jinan University, \\ Guangzhou 510632, People's Republic of China \\ ${ }^{2}$ School of Physics, Sun Yat-Sen University, Guangzhou 510275, People's Republic of China \\ ${ }^{3}$ CAS Key Laboratory of Theoretical Physics, Institute of Theoretical Physics, \\ Chinese Academy of Sciences, Beijing 100190, People's Republic of China \\ ${ }^{4}$ School of Physical Sciences, University of Chinese Academy of Sciences, \\ Beijing 100049, People's Republic of China
}

(Received 6 May 2021; accepted 21 June 2021; published 7 July 2021)

\begin{abstract}
We consider a scenario where light bino is the next-to-lightest supersymmetric particle (NLSP) and gravitino/axino is the lightest superysmmetric particle (LSP). For a bino mass less than or around hundred $\mathrm{GeV}$, it can be pair produced at the future lepton colliders through $t$-channel slepton exchange, subsequently decaying into a gravitino/axino plus a photon. We study the prospects to look for such binos at the future colliders and find that a bino mass around $100 \mathrm{GeV}$ can be probed at the $2 \sigma(5 \sigma)$ level for a slepton below $2 \mathrm{TeV}(1.5 \mathrm{TeV})$ with a luminosity $5.6 \mathrm{ab}^{-1}$. For a bino mass around $10 \mathrm{GeV}$, a slepton mass less than $4.5 \mathrm{TeV}(3.5 \mathrm{TeV})$ can be probed at the $2 \sigma(5 \sigma)$ level, which is much beyond the reach of the LHC for direct slepton searches.
\end{abstract}

DOI: 10.1103/PhysRevD.104.015009

\section{INTRODUCTION}

Despite no experimental evidence, supersymmetry remains one of the most compelling scenarios beyond the standard model. It not only alleviates the fine-tuning of Higgs mass contrasting to the fundamental scale, but also predicts the unification of the gauge couplings and provides a viable dark matter candidate. Currently the large hadron colliders (LHC) already set very strong limits on the mass scale of SUSY partners. For example, the gluino and squarks should be beyond $1-2 \mathrm{TeV}[1,2]$ and the limits on electroweakinos and sleptons are around few hundreds $\mathrm{GeV}$ depending on the assumptions [3,4]. For a degenerate spectrum such as Higgsinos or winos, the strongest bounds are from the LEP and their masses should be larger than around $100 \mathrm{GeV}$ [5]. However, there remains a possibility that a bino could be as light as few tens of GeV. Given the design of a future lepton colliders [6-8], it provides a good opportunity to look for such a light bino.

If a bino is the lightest supersymmetric particle (LSP) as well as the dark matter candidate, it usually has

\footnotetext{
* Corresponding author. mczhang@jnu.edu.cn

These authors share first authorship.

Published by the American Physical Society under the terms of the Creative Commons Attribution 4.0 International license. Further distribution of this work must maintain attribution to the author(s) and the published article's title, journal citation, and DOI. Funded by SCOAP ${ }^{3}$.
}

overabundance for a light bino below $100 \mathrm{GeV}$. For a well-tempered bino-Higgsino mixing dark matter, it is already excluded by dark matter direct searches for the light mass region [9-11]. Therefore it is reasonable to consider that a light bino might be the next-to-lightest supersymmetric particle (NLSP), while an additional particle is taken as the LSP. One of the natural LSP candidates is the gravitino, which could be much lighter than $\mathrm{GeV}$ for a low SUSY breaking scale such as in the gauge mediation models $[12,13]$. Another possibility is that the LSP could be the axino, which is predicted by the breaking of PecceiQuinn symmetry to solve the strong $C P$ problem in the framework of supersymmetry $[14,15]$. In both cases, the bino could probably decay into a photon and a LSP, providing a good signal at colliders. Such searches were already performed at the LHC where the gluino pair production sequentially decaying into neutralino and two quarks and the neutralino later decays into a photon and a gravitino [16]. Here we consider the bino pair production at lepton colliders through exchanging a t-channel slepton, and then the bino decays in the channel $\tilde{B} \rightarrow \tilde{G} / \tilde{a}+\gamma .{ }^{1}$ The typical signal is two photons plus large missing energy. This events has been collected by the L3 detector at LEP with luminosity $619 \mathrm{pb}^{-1}$ and center-of-mass energy

\footnotetext{
${ }^{1}$ There are also $\tilde{B} \rightarrow \tilde{G} / \tilde{a}+h / Z$ decay channels. However, for the bino mass region we are interested in these decay channels are suppressed by phase space, therefore we just ignore them and consider the branching ratio of $\tilde{B} \rightarrow \tilde{G} / \tilde{a}+\gamma$ is $100 \%$.
} 
between $189 \mathrm{GeV}$ and $209 \mathrm{GeV}$ [17]. The result is consistent with the SM, and selectron can be excluded up to several hundred $\mathrm{GeV}$ scale, depending on bino mass. In this work, we will analyze the kinematics of these two photons plus missing energy signal in detail, and propose suitable kinematical variables to distinguish our SUSY signal from the SM background. Through a reliable simulation, we expect such a signal could be detected at future lepton colliders (e.g., CEPC) for a selectron mass less than a few $\mathrm{TeV}$, and it would be much beyond the slepton searches at the LHC and LEP.

This paper is organized as follows: in Sec. II we discuss the related collider analysis including the kinetic variables for cutting. In Sec. III we present our numerical results and we draw our conclusion in Sec. IV.

\section{COLLIDER ANALYSIS}

In this section we analyse the collider signatures of the bino pair production at the lepton colliders. As a benchmark model, we study the scenario of gravitino as the LSP. The signal we consider is $e^{+} e^{-} \rightarrow \tilde{B} \tilde{B} \rightarrow \gamma \gamma \tilde{G} \tilde{G}$. The main SM background is $e^{+} e^{-} \rightarrow \gamma \gamma \nu \bar{\nu}$, with $Z$ or $W^{ \pm}$boson being the internal particles. In Fig. 1 we show the Feynman diagrams to illustrate our signal and background processes.

Both the signal and backgrounds are induced by electroweak interaction, but the signal is suppressed by the $\mathrm{TeV}$ scale selectron and thus the production cross section is much smaller. In the following we calculate the cross section of bino pair production at an electron-positron collider with center-of-mass energy $\sqrt{s}$. For $e_{R}^{-} e_{L}^{+} \rightarrow \tilde{B} \tilde{B}$, where the bino production is mediated by $\tilde{e}_{R}$, the corresponding total cross section is

$$
\begin{aligned}
\sigma_{\mathrm{tot}}^{R}= & \frac{g_{1}^{4} Y_{e_{R}}^{4}}{8 \pi s^{2}}\left[4 E|\vec{k}|-\left(2 m_{\tilde{e}_{R}}^{2}-2 m_{\tilde{\tilde{B}}}^{2} \frac{2\left(m_{\tilde{e}_{R}}^{2}-m_{\tilde{B}}^{2}\right)}{s+2\left(m_{e_{R}}^{2}-m_{\tilde{B}^{2}}^{2}\right)}\right)\right. \\
& \left.\times \log \frac{\left(2 E(E+|\vec{k}|)+m_{\tilde{e}_{R}}^{2}-m_{\tilde{B}}^{2}\right)^{2}}{4 E^{2} m_{\tilde{e}_{R}}^{2}+\left(m_{\tilde{e}_{R}}^{2}-m_{\tilde{B}}^{2}\right)^{2}}\right] \\
& +\frac{g_{1}^{4} Y_{e_{R}}^{4}}{8 \pi s^{2}}\left(m_{\tilde{e}_{R}}^{2}-m_{\tilde{B}^{2}}^{2}\right)^{2} \frac{4 E(E+|\vec{k}|)}{4 E^{2} m_{\tilde{e}_{R}}^{2}+\left(m_{\tilde{e}_{R}}^{2}-m_{\tilde{B}}^{2}\right)^{2}} .
\end{aligned}
$$

For $e_{L}^{-} e_{R}^{+} \rightarrow \tilde{B} \tilde{B}$ process which is mediated by $\tilde{e}_{L}$, the corresponding total cross section is

$$
\begin{aligned}
\sigma_{\text {tot }}^{L}= & \frac{g_{1}^{4} Y_{e_{L}}^{4}}{8 \pi s^{2}}\left[4 E|\vec{k}|-\left(2 m_{\tilde{e}_{L}}^{2}-2 m_{\tilde{\tilde{B}}}^{2} \frac{2\left(m_{\tilde{e}_{L}}^{2}-m_{\tilde{B}}^{2}\right)}{s+2\left(m_{e_{L}}^{2}-m_{\tilde{B}^{2}}^{2}\right)}\right)\right. \\
& \left.\times \log \frac{\left(2 E(E+|\vec{k}|)+m_{\tilde{e}_{L}}^{2}-m_{\tilde{B}}^{2}\right)^{2}}{4 E^{2} m_{\tilde{e}_{L}}^{2}+\left(m_{\tilde{e}_{L}}^{2}-m_{\tilde{B}}^{2}\right)^{2}}\right] \\
& +\frac{g_{1}^{4} Y_{e_{L}}^{4}}{8 \pi s^{2}}\left(m_{\tilde{e}_{L}}^{2}-m_{\tilde{B}^{2}}^{2}\right)^{2} \frac{4 E(E+|\vec{k}|)}{4 E^{2} m_{\tilde{e}_{L}}^{2}+\left(m_{\tilde{e}_{L}}^{2}-m_{\tilde{B}}^{2}\right)^{2}}
\end{aligned}
$$

In the above expressions, $k^{\mu}=(E, \vec{k})$ is the 4-momentum of one of the binos and $s=4 E^{2}$ is the central energy square. We have set $m_{e}=0$. If $\tilde{e}_{R}$ and $\tilde{e}_{L}$ have the same masses, then the ratio between $\sigma_{\text {tot }}^{R}$ and $\sigma_{\text {tot }}^{L}$ is

$$
\frac{\sigma_{\mathrm{tot}}^{L}}{\sigma_{\mathrm{tot}}^{R}}=\frac{Y_{e_{L}}^{4}}{Y_{e_{R}}^{4}}=\frac{1}{16}
$$

For simplicity, in the following we assume the masses of $\tilde{e}_{R}$ and $\tilde{e}_{L}$ are equal and define $m_{\tilde{e}} \equiv m_{\tilde{e}_{R}}=m_{\tilde{e}_{L}}$. Then the total cross section can be simplified to the following formula if selectrons are heavy enough:

$\sigma_{\mathrm{tot}}\left(e^{+} e^{-} \rightarrow \tilde{B} \tilde{B}\right) \approx \frac{g_{1}^{4}}{48 \pi}\left(Y_{e_{L}}^{4}+Y_{e_{R}}^{4}\right) \frac{s}{m_{\tilde{e}}^{4}}\left[1-\left(\frac{m_{\tilde{B}}}{E}\right)^{2}\right]^{3 / 2}$.

In Fig. 2 we show the cross sections of $e^{+} e^{-} \rightarrow \tilde{B} \tilde{B}$ and the SM background $e^{+} e^{-} \rightarrow \gamma \gamma \nu \bar{\nu}$ for $\sqrt{s}=240 \mathrm{GeV}$. Here we use MADGRAPH5 [18] to calculate the cross section of $e^{+} e^{-} \rightarrow \gamma \gamma \nu \bar{\nu}$. Due to the soft and collinear divergence when photons are radiated from the initial state electron and positron, we require the transverse momentums of photons in the final state to be larger than $10 \mathrm{GeV}$. It is clear that a TeV-scale $m_{\tilde{e}}$ highly suppresses the cross section of the signal. To improve the direct search sensitivity, additional cuts are needed. The final state of the signal and background processes are extremely simple and clear, only two photons and missing momentum. What we

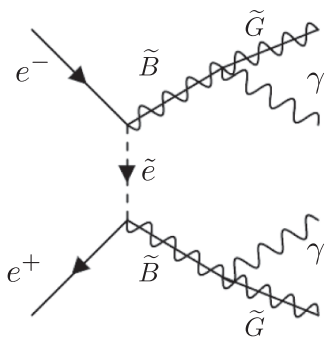

(a)

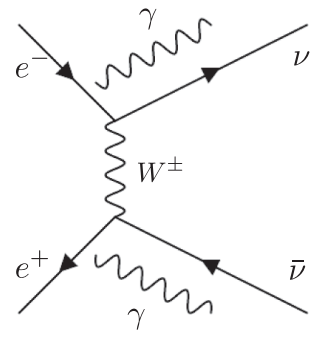

(b)

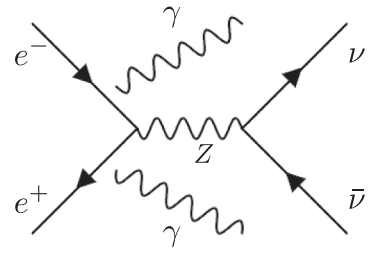

(c)

FIG. 1. (a) Feynman diagram of signal process; (b) Feynman diagrams of background process with t-channel $W$ boson and (c) Feynman diagrams of background process with s-channel $Z$ boson, where two photons can couple to any charged particles. 


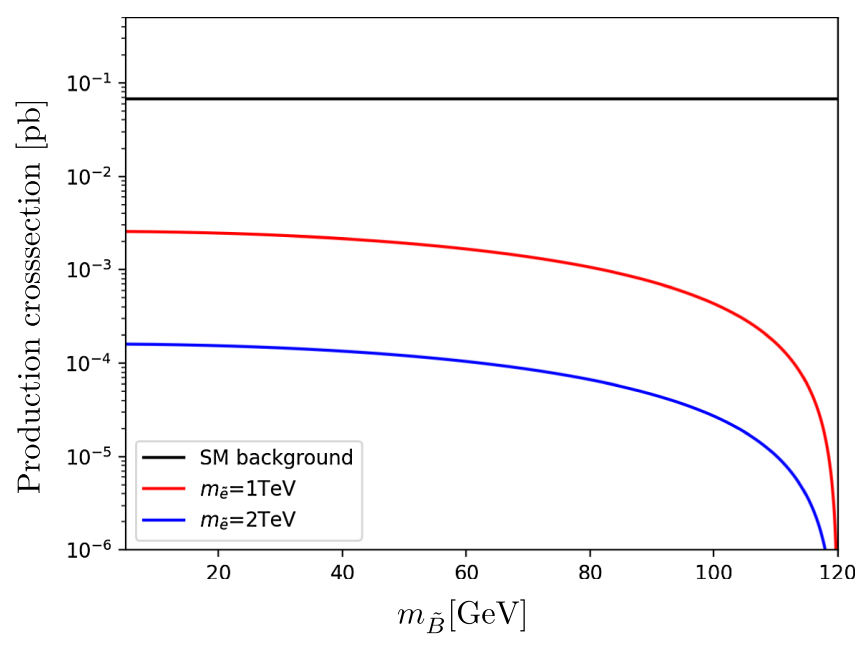

FIG. 2. The black line is the cross section of $\sigma\left(e^{+} e^{-} \rightarrow \gamma \gamma \nu \bar{\nu}\right)$ in the SM, with photon $p_{\mathrm{T}}>10 \mathrm{GeV}$. The red and blue curves are the cross section of $\sigma\left(e^{+} e^{-} \rightarrow \tilde{B} \tilde{B}\right)$ with $m_{\tilde{e}}$ being $1 \mathrm{TeV}$ and $2 \mathrm{TeV}$, respectively. The center-of-mass energy is fixed to $240 \mathrm{GeV}$.

can directly measure are the 4-momentums of the two final state photons:

$$
\begin{aligned}
& p^{\gamma 1}=\left(E^{\gamma 1}, p_{x}^{\gamma 1}, p_{y}^{\gamma 1}, p_{z}^{\gamma 1}\right) \\
& p^{\gamma 2}=\left(E^{\gamma 2}, p_{x}^{\gamma 2}, p_{y}^{\gamma 2}, p_{z}^{\gamma 2}\right)
\end{aligned}
$$

These 8 components will be the inputs of our analysis and all the kinetic variables will be constructed from them. For Monte Carlo simulation, we use MADGRAPH5 [18] to generate the parton-level signal and background events. QED shower, which can happen when the energy of charged particle is much larger than its mass, is performed by PYTHIA [19]. Considering the detector effect, we perform a Gaussian smearing on the photon energy measurement with uncertainty $3 \%$ [20].

\section{A. Variable-1: Recoiled mass}

The first kinetic variable we will use is the recoiled mass, which has been proven to be a useful variable in Higgs measurements at lepton colliders [21]. Unlike the hadron collider with undermined initial-state parton 4-momentum, the 4-momentums of the initial-state electron and positron are known. For a lepton collider with center-of-mass energy square $s$, the signal contains two photons in the final state. Thus from the 4-momentum conservation we have

$$
(\sqrt{s}, 0,0,0)=p^{\gamma 1}+p^{\gamma 2}+\not p
$$

By knowing $p^{\gamma 1}$ and $p^{\gamma 2}$, we can construct the recoiled mass as

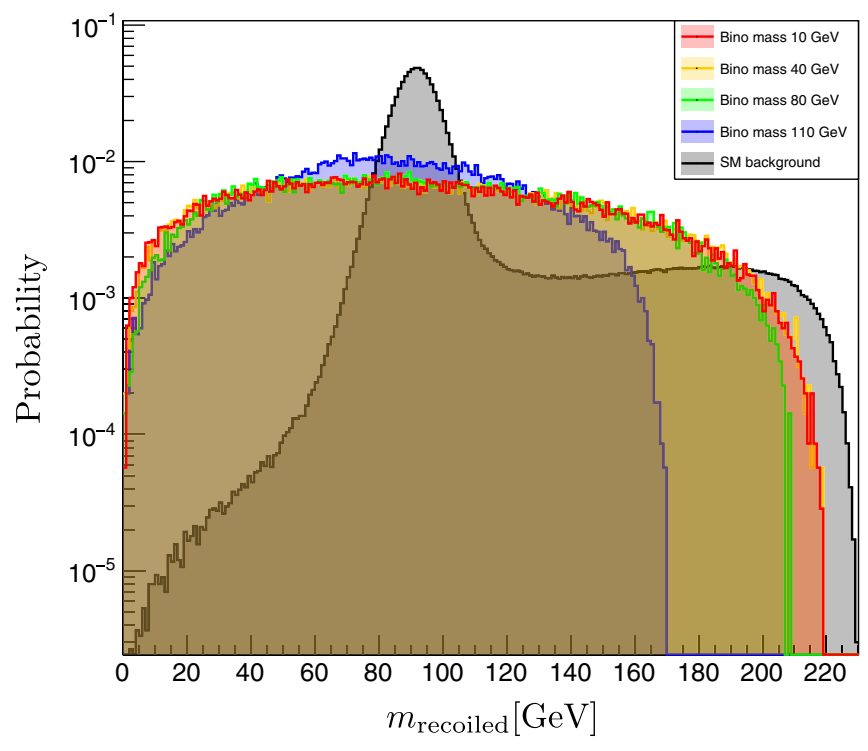

FIG. 3. Recoiled mass distributions for the signal and SM background processes with different bino masses.

$$
m_{\mathrm{rec}}=\sqrt{\left((\sqrt{s}, 0,0,0)-p^{\gamma 1}-p^{\gamma 2}\right)^{2}}
$$

We expect this recoiled mass to peak around the $Z$ boson mass for the SM background process because the SM background is dominated by the s-channel $Z$ boson process. And for our signal process, the distribution of $m_{\text {rec }}$ should be much more flat because the missing momentum in signal process comes from two gravitinos of two branches.

In Fig. 3 we present the recoiled mass distribution for the SM background and signal process with different bino masses. As expected, the SM distribution peaks around $90 \mathrm{GeV}$, which comes from the s-channel $Z$ boson. The recoiled mass from t-channel $W$ boson tends to have a larger value, and the distribution extends smoothly to the kinetically allowed region. On the contrary, the recoiled mass of signal process is basically evenly distributed. Therefore, the background can be hugely suppressed by requiring an upper limit of $m_{\text {recoiled }}$.

\section{B. Variable-2: Reconstructed mass}

Since there are two decay branches with invisible particles in the final states, the mass of bino can not be easily reconstructed. At hadron collider, one can use the variables like MT2 [22-25] to discriminate signal from SM background. At lepton collider, momentums in longitudinal direction can also be measured, and thus the kinematics are different with hadron collider. Here we propose a kinetic variable which can reflect the bino mass through its distribution.

In addition to the 8 known values (4-momentums of two photons), there are 8 unknown values which come from invisible gravitinos: 


$$
\begin{aligned}
& p^{\tilde{G} 1}=\left(E^{\tilde{G} 1}, p_{x}^{\tilde{G} 1}, p_{y}^{\tilde{G} 1}, p_{z}^{\tilde{G} 1}\right) \\
& p^{\tilde{G} 2}=\left(E^{\tilde{G} 2}, p_{x}^{\tilde{G} 2}, p_{y}^{\tilde{G} 2}, p_{z}^{\tilde{G} 2}\right) .
\end{aligned}
$$

The missing momentum is composed by these two invisible momentums. For a lepton collider, we know the 4-momentums of initial states. Thus the missing momentum can be calculated from the momentums of two photons, and we obtain 4 constraint equations for 8 unknown values:

$$
\begin{gathered}
E^{\tilde{G} 1}+E^{\tilde{G} 2}=\sqrt{s}-E^{\gamma 1}-E^{\gamma 2} \\
p_{x}^{\tilde{G} 1}+p_{x}^{\tilde{G} 2}=-p_{x}^{\gamma 1}-p_{x}^{\gamma 2} \\
p_{y}^{\tilde{G} 1}+p_{y}^{\tilde{G} 2}=-p_{y}^{\gamma 1}-p_{y}^{\gamma 2} \\
p_{z}^{\tilde{G} 1}+p_{z}^{\tilde{G} 2}=-p_{z}^{\gamma 1}-p_{z}^{\gamma 2}
\end{gathered}
$$

with $\sqrt{s}$ being the center-of-mass energy of the $e^{+} e^{-}$ collider. By using these four constraint conditions, the number of unknown values decrease from 8 to 4 . We can further limit those unknown values by using the mass of gravitino $m_{\tilde{G}}$, which is smaller than GeV. Such a tiny mass can be treated as zero for our collider kinematic analysis. Then the on-shell conditions give two more constraint equations:

$$
\begin{aligned}
& \left(E^{\tilde{G} 1}\right)^{2}=\left(p_{x}^{\tilde{G} 1}\right)^{2}+\left(p_{y}^{\tilde{G} 1}\right)^{2}+\left(p_{z}^{\tilde{G} 1}\right)^{2} \\
& \left(E^{\tilde{G} 2}\right)^{2}=\left(p_{x}^{\tilde{G} 2}\right)^{2}+\left(p_{y}^{\tilde{G} 2}\right)^{2}+\left(p_{z}^{\tilde{G} 2}\right)^{2}
\end{aligned}
$$

and the number of unknown values decrease from 4 to 2 . And we can reexpress the 4-momentums of $\tilde{G} 1$ and $\tilde{G} 2$ as

$$
\begin{gathered}
p^{\tilde{G} 1}=E^{\tilde{G} 1}\left(1, \vec{e}_{1}\right) \\
p^{\tilde{G} 2}=E^{\tilde{G} 2}\left(1, \vec{e}_{2}\right) .
\end{gathered}
$$

Here we use the unit 3-vectors $\vec{e}_{1}$ and $\vec{e}_{2}$ to represent the moving directions of $\tilde{G} 1$ and $\tilde{G} 2$, respectively.

The last advantage of a lepton collider that we are going to use is that the two binos must be generated with the same speed. Thus the energies of these two binos must be equal. Combining with energy conservation, we obtain two linear equations that can be used to fix $E^{\tilde{G} 1}$ and $E^{\tilde{G} 2}$ :

$$
\left\{\begin{array} { l } 
{ E ^ { \tilde { G } 1 } + E ^ { \tilde { G } 2 } = \sqrt { s } - E ^ { \gamma 1 } - E ^ { \gamma 2 } } \\
{ E ^ { \tilde { G } 1 } + E ^ { \gamma 1 } = E ^ { \tilde { G } 2 } + E ^ { \gamma 2 } }
\end{array} \Rightarrow \left\{\begin{array}{l}
E^{\tilde{G} 1}=\frac{\sqrt{s}}{2}-E^{\gamma 1} \\
E^{\tilde{G} 2}=\frac{\sqrt{s}}{2}-E^{\gamma 2}
\end{array}\right.\right.
$$

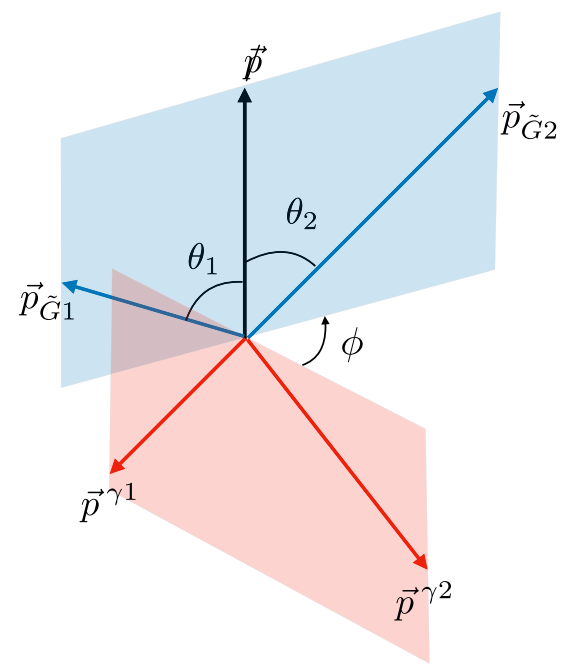

FIG. 4. Geometric relations between $\vec{p}_{\tilde{G} 1}, \vec{p}_{\tilde{G} 2}$ and the 3 measured momentums. $\vec{p}_{\tilde{G} 1}, \vec{p}_{\tilde{G} 2}$, and $\vec{p}$ are in a plane, while $\vec{p}^{\gamma 1}, \vec{p}^{\gamma 2}$, and $\vec{p}$ are in another plane. The angle $\phi$ between the two planes can not be fixed by measurable quantities.

Now we only have one unknown value in this kinematic system. In the following we explain what is the last ambiguity. We label the 3-momentums of the two binos and total missing momentum as $\vec{p}_{\tilde{G} 1}, \vec{p}_{\tilde{G} 2}$, and $\vec{p}$ :

$$
\begin{aligned}
\vec{p}_{\tilde{G} 1} & =E^{\tilde{G} 1} \vec{e}_{1}, \quad \vec{p}_{\tilde{G} 2}=E^{\tilde{G} 2} \vec{e}_{2}, \\
\vec{p} & =\left(-p_{x}^{\gamma 1}-p_{x}^{\gamma 2},-p_{y}^{\gamma 1}-p_{y}^{\gamma 2},-p_{z}^{\gamma 1}-p_{z}^{\gamma 2}\right)
\end{aligned}
$$

We already knew $\vec{p}$ and the lengthes of $\vec{p}_{\tilde{G} 1}$ and $\vec{p}_{\tilde{G} 2}$. Because $\vec{p}_{\tilde{G} 1}+\vec{p}_{\tilde{G} 2}=\vec{p}$, so these 3 vectors must be in the same plane. On the other hand, the total 3-momentum of final states should be zero, so $\vec{p}^{\gamma 1}, \vec{p}^{\gamma 2}$, and $\vec{p}$ are in another plane. For illustration, we show their geometry in Fig. 4. Fixing the lengthes of $\vec{p}_{\tilde{G} 1}$ and $\vec{p}_{\tilde{G} 2}$, we can determine the angle between them and $\vec{p}$ (noted as $\theta_{1}$ and $\theta_{2}$ in Fig. 4). But the angle between the two different planes (noted as $\phi$ in Fig. 4), cannot be determined by input values. So the value of $\phi$ is the last variable that we cannot fix.

The value range of $\phi$ is 0 to $2 \pi$. We can calculate the trial mass of bino (noted as $m_{\tilde{B} 1}^{*}$ and $m_{\tilde{B} 2}^{*}$ ) at each branch as a function of $\phi$ :

$$
\begin{aligned}
m_{\tilde{B} 1}^{*}(\phi) & =\sqrt{\left(E^{\tilde{G} 1}+E^{\gamma 1}\right)^{2}-\left(\vec{p}_{\tilde{G} 1}+\vec{p}^{\gamma 1}\right)^{2}} \\
& =\sqrt{2 E^{\tilde{G} 1} E^{\gamma 1}-2 \vec{p}_{\tilde{G} 1} \cdot \vec{p}^{\gamma 1}} \\
m_{\tilde{B} 2}^{*}(\phi) & =\sqrt{\left(E^{\tilde{G} 2}+E^{\gamma 2}\right)^{2}-\left(\vec{p}_{\tilde{G} 2}+\vec{p}^{\gamma 2}\right)^{2}} \\
& =\sqrt{2 E^{\tilde{G} 2} E^{\gamma 2}-2 \vec{p}_{\tilde{G} 2} \cdot \vec{p}^{\gamma 2}}
\end{aligned}
$$

where $\vec{p}_{\tilde{G} 1}$ and $\vec{p}_{\tilde{G} 2}$ are functions of $\phi$. Furthermore, $m_{\tilde{B} 1}^{*}(\phi)=m_{\tilde{B} 2}^{*}(\phi)$ holds for any value of $\phi$ because this 


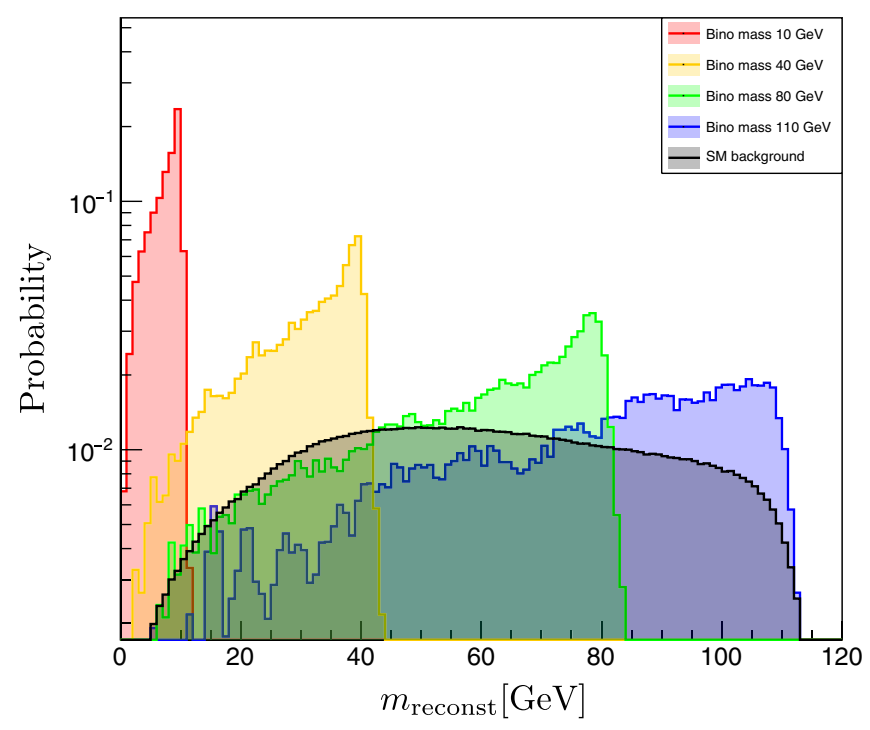

FIG. 5. Reconstructed mass distributions for the signal and SM background processes with different bino masses.

condition has been used in Eq. (19). In the following we denote both $m_{\tilde{B} 1}^{*}(\phi)$ and $m_{\tilde{B} 2}^{*}(\phi)$ indiscriminately as $m_{\tilde{B}}^{*}(\phi) . m_{\tilde{B}}^{*}(\phi)$ can be minimized with a certain value of $\phi$. This minimum value is equal to the real bino mass only when all the momentums in Fig. 4 are in a same plane, otherwise the minimum value of $m_{\widetilde{B}}^{*}(\phi)$ is smaller than the real bino mass. Thus we define the reconstructed mass as the minimum value of $m_{\tilde{B}}^{*}(\phi)$ :

$$
m_{\text {reconst }}=\min _{\phi \in(0,2 \pi)} m_{\tilde{B}}^{*}(\phi)
$$

For the signal process, $m_{\text {reconst }}$ cannot exceed the real bino mass. In Fig. 5 we present the distributions of $m_{\text {reconst }}$ for the SM background and signal process with different bino masses. It can be seen that for the signal process, $m_{\text {reconst }}$ basically distribute in the region that smaller than the real bino mass. The events that exceed the bino mass limit come from the uncertainty in the photon energy measurement. The distribution of $m_{\text {reconst }}$ peaks when it is close to the real bino mass. On the other hand the reconstructed mass for the background process is basically evenly distributed. Thus a simple mass window cut on $m_{\text {reconst }}$ can help us to enhance the search sensitivity.

\section{Variable-3: Energy balance}

To improve the sensitivity in the heavy bino region, we introduce another variable energy balance which is defined as

$$
\mathcal{A}_{\text {balance }}=\frac{\left|E^{\gamma 1}-E^{\gamma 2}\right|}{\left|E^{\gamma 1}+E^{\gamma 2}\right|}
$$

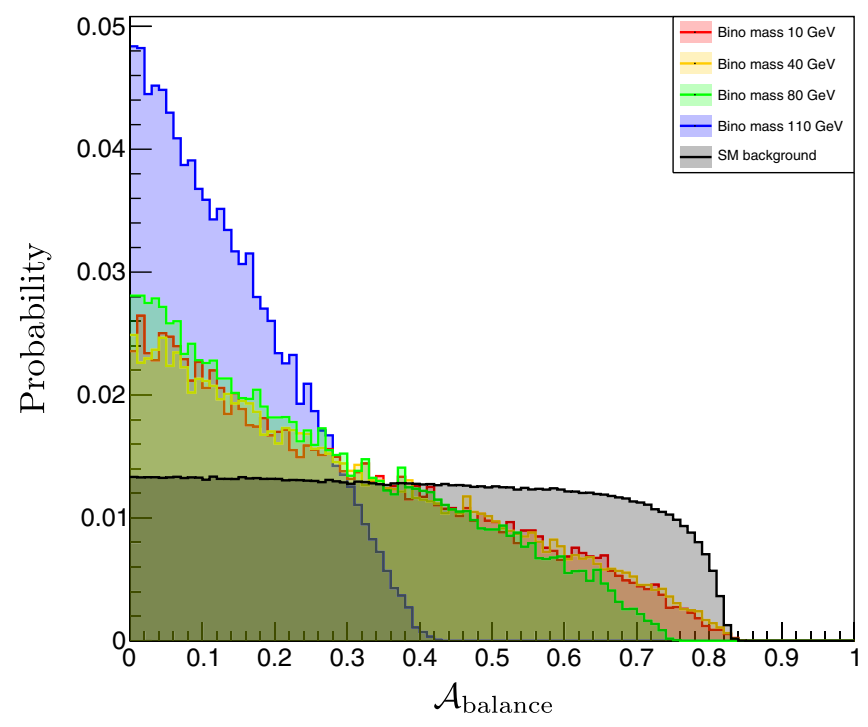

FIG. 6. Energy balance distributions for the signal and SM background processes with different bino masses.

This variable can be very useful in signal and background discrimination when bino is heavy. ${ }^{2}$ In this case, the bino is not so boosted and the energy of each final-state photon is around the half of bino mass. Then the value of $\mathcal{A}_{\text {balance }}$ will be quite small when bino is heavy. On the contrary, if bino is light, the energy of each final-state photon is quite random (depending on the decay direction) and the distribution of $\mathcal{A}_{\text {balance }}$ will be more uniform. For the background process, the distribution should also be flat. In Fig. 6 we present the distributions of $\mathcal{A}_{\text {balance }}$ for the SM background and signal processes with different bino masses.

\section{NUMERICAL RESULTS}

In this section we use the three variables defined in the preceding section to perform cuts for our signal and backgrounds. It should be noted that the final state kinetic distributions are almost not relevant to the mass of selectron $m_{\tilde{e}}$ which only rescales the total cross section of the signal process roughly by a factor $1 / m_{\tilde{e}}^{4}$. To illustrate the performance of the cut-flow, we will fix $m_{\tilde{e}}$ to $1 \mathrm{TeV}$ and vary $m_{\tilde{B}}=10 \mathrm{GeV}, 40 \mathrm{GeV}, 80 \mathrm{GeV}, 110 \mathrm{GeV}$ as our benchmark points. The final results will be given in the end of this section. For future lepton colliders, we focus on a center-of-mass energy of $240 \mathrm{GeV}$ and an integral luminosity of $5.6 \mathrm{ab}^{-1}$.

The following cuts are imposed to enhance the search sensitivity:

(i) Recoiled mass $m_{\text {recoiled }}$ is required to be smaller than $40 \mathrm{GeV}$. The reason for this requirement can be seen

\footnotetext{
${ }^{2}$ Similar variables also used in other lepton collider studies, e.g., [26].
} 
TABLE I. The cut flow for the SM background and 4 benchmark points of the signal. For the lepton collider we set the center-of-mass energy to be $240 \mathrm{GeV}$ and the integral luminosity to be $5.6 \mathrm{ab}^{-1}$. For different benchmark points, we perform different mass window cuts on $m_{\text {reconst }}$.

\begin{tabular}{|c|c|c|c|c|c|}
\hline & BKG & $m_{\tilde{B}}=10 \mathrm{GeV}$ & $m_{\tilde{B}}=40 \mathrm{GeV}$ & $m_{\tilde{B}}=80 \mathrm{GeV}$ & $m_{\tilde{B}}=110 \mathrm{GeV}$ \\
\hline Photon $p_{\mathrm{T}}>10 \mathrm{GeV}$ & 376,880 & 10,550 & 9,427 & 5,529 & 879.5 \\
\hline$m_{\text {recoiled }}<40 \mathrm{GeV}$ & 258.8 & 2,187 & 1,837 & 838.1 & 105.9 \\
\hline $\mathcal{A}_{\text {balance }}<0.3$ & 74.9 & 1,578 & 1,342 & 598.3 & 89.5 \\
\hline$m_{\text {reconst }} \in[0,13] \mathrm{GeV}$ & 1.6 & 1,571 & $\ldots$ & $\cdots$ & $\cdots$ \\
\hline$m_{\text {reconst }} \in[30,43] \mathrm{GeV}$ & 5.7 & $\cdots$ & 667.6 & $\cdots$ & $\cdots$ \\
\hline$m_{\text {reconst }} \in[70,83] \mathrm{GeV}$ & 6.8 & $\cdots$ & $\cdots$ & 191.3 & $\cdots$ \\
\hline$m_{\text {reconst }} \in[100,113] \mathrm{GeV}$ & 20.4 & $\cdots$ & $\cdots$ & $\cdots$ & 54.2 \\
\hline $\mathcal{S} / \mathcal{B}$ & $\cdots$ & 981.9 & 117.1 & 28.1 & 2.7 \\
\hline Significance & $\cdots$ & 136.1 & 71.3 & 30.9 & 9.2 \\
\hline
\end{tabular}

from Fig. 3. It is clear that the distribution of $m_{\text {recoiled }}$ for the background process rapidly decrease in the low $m_{\text {recoiled }}$ region. Thus removing the event with large $m_{\text {recoiled }}$ will suppress the background hugely.

(ii) Energy balance $\mathcal{A}_{\text {balance }}$ is required to be smaller than 0.3. As can be seen from Fig. 6, the requirement helps to enhance the search sensitivity when bino is heavy.

(iii) Mass window cut is performed on the reconstructed mass $m_{\text {reconst }}$ :

$$
m_{\tilde{B}}-10 \mathrm{GeV} \leqslant m_{\text {reconst }} \leqslant m_{\tilde{B}}+3 \mathrm{GeV}
$$

This mass window cut depends on the bino mass. The upper and lower limits of mass window are chosen to optimize the search sensitivity.

In Table I we present the cut flows for the SM background and our benchmark points. It shows that the $m_{\text {recoiled }}<40 \mathrm{GeV}$ cut reduce the SM background by three orders of magnitude, but only reduce the signal by one order of magnitude. The cuts of energy balance and mass window further reduce the SM background by about one or two orders of magnitude, without hurting the signal too much. We use $\mathcal{S}$ and $\mathcal{B}$ to represent the number of signal and background events after the cut flow, respectively. Then the statistical significance is evaluated by Poisson formula $^{3}$

$$
\text { Significance }=\sqrt{2\left[(\mathcal{S}+\mathcal{B}) \ln \left(1+\frac{\mathcal{S}}{\mathcal{B}}\right)-\mathcal{S}\right]} .
$$

For observation or exclusion, we require $\mathcal{S} / \mathcal{B}$ should be larger than $10 \%$ and the significance should be

\footnotetext{
${ }^{3}$ More advanced discussion about statistical significance see [27].
}

larger than 5 or 2 . In the last two rows of Table I we show $\mathcal{S} / \mathcal{B}$ and the significance for each benchmark point. Therefore, for all 4 benchmark points the statistical significances are much larger than $5 \sigma$ for a slepton mass around $1 \mathrm{TeV}$.

At the end, we can obtain the $2 \sigma$ exclusion limit (5 $\sigma$ observation limit) on $m_{\tilde{e}}-m_{\tilde{B}}$ plane by requiring $\mathcal{S} / \mathcal{B}>0.1$ and Significance $>2$ (Significance $>5$ ). For each $m_{\tilde{B}}$ we obtain the signal acceptance by performing our cut-flow. The signal acceptance as a function of $m_{\tilde{B}}$ is given in Fig. 7 (left). We also present the acceptance of SM background as function of $m_{\tilde{B}}$ in Fig. 7 (left). ${ }^{4}$ By using the acceptance information and the cross section given by Eq. (4), we can easily calculate the significance for each spectrum $\left(m_{\tilde{e}}, m_{\tilde{B}}\right)$, and thus find out the $2 \sigma$ exclusion and $5 \sigma$ observation limits on the $m_{\tilde{e}}-m_{\tilde{B}}$ plane. We show our final results in Fig. 7 (right). It tells that a future lepton collider with an integral luminosity $5.6 \mathrm{ab}^{-1}$ and center-ofmass energy $240 \mathrm{GeV}$ can exclude a bino below about $10 \mathrm{GeV}$ for selectron less than $4.5 \mathrm{TeV}$, or a bino below about $100 \mathrm{GeV}$ for a selectron less than $2 \mathrm{TeV}$, which is much beyond the reach of the LHC for direct slepton searches.

Before finishing this section, we give a brief comment on selectron and bino search on LEP [17]. In Ref. [17], single or multiphoton events with missing energy are collected. The final observed events number are in agreement with the SM expectations, and thus an exclusion limit on $m_{\tilde{e}}$ and $m_{\tilde{B}}$ is obtained. However, a dedicated cut-flow which is designed to enhance the selectron and bino search sensitivity is not performed in [17]. Thus we expect that a more stringent LEP limit on $m_{\tilde{e}}$ and $m_{\tilde{B}}$ can be obtained by reanalyzing the old LEP data with the help of the variables we used in this work.

\footnotetext{
${ }^{4}$ Mass window cut depends on the value of $m_{\tilde{B}}$.
} 

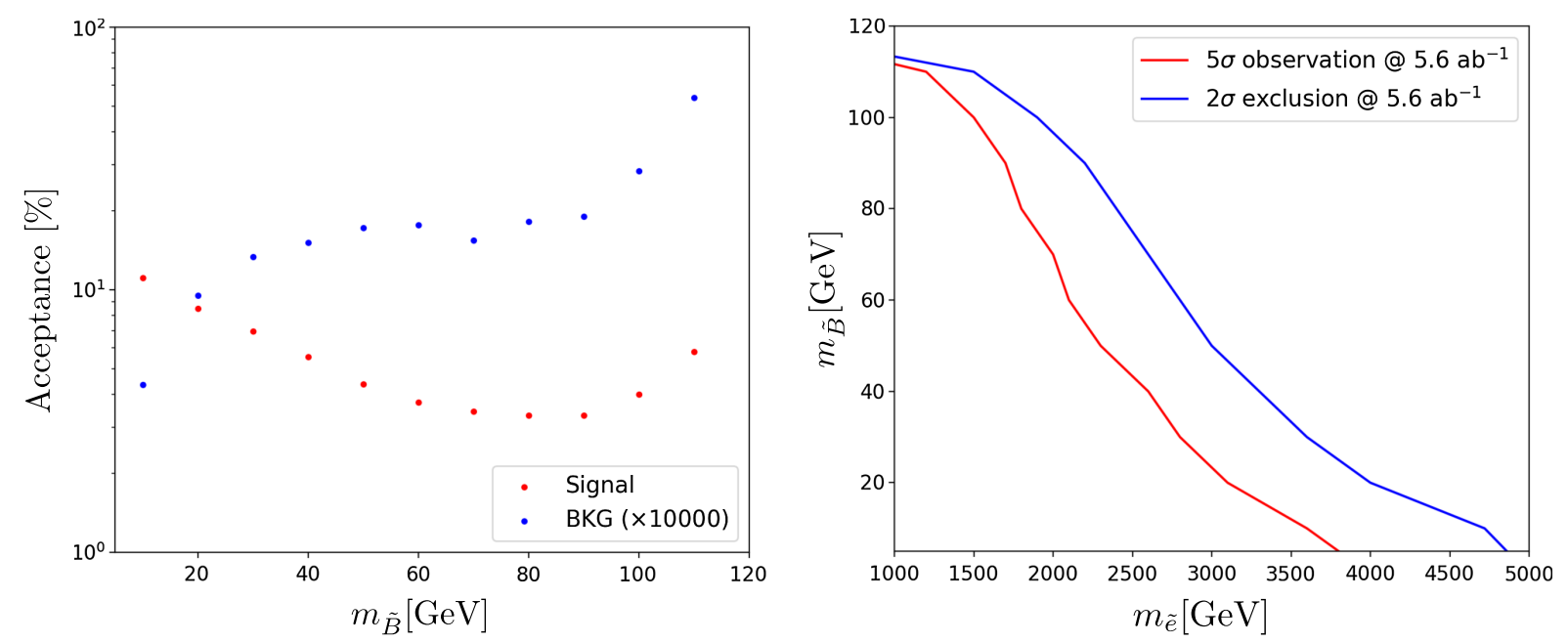

FIG. 7. Left: acceptance of signal and background processes as functions of $m_{\tilde{B}}$. Here the acceptance of background process has been multiplied by 10,000 . Right: $2 \sigma$ exclusion and $5 \sigma$ observation limits on the $m_{\tilde{e}}-m_{\tilde{B}}$ plane at a future lepton collider running with an integral luminosity $5.6 \mathrm{ab}^{-1}$ and center-of-mass energy $240 \mathrm{GeV}$. Regions below the curves are observable or excluded.

\section{CONCLUSION}

The bino is the only supersymmetric partner whose mass could be less than a hundred $\mathrm{GeV}$. The future lepton colliders could provide a good opportunity to probe such a light particle. We considered a scenario where a light bino is the next-tolightest superysmmetric particle (NLSP) and the gravition/ axino is the lightest superysmmetric particle (LSP). Then the bino can probably decay into a photon plus the LSP. We studied the bino pair production at the future colliders and found that a bino mass around $100 \mathrm{GeV}$ can be probed at the $2 \sigma(5 \sigma)$ level for a slepton below $2 \mathrm{TeV}(1.5 \mathrm{TeV})$ with a luminosity $5.6 \mathrm{ab}^{-1}$. For a bino mass around $10 \mathrm{GeV}$, a slepton mass less than $4.5 \mathrm{TeV}(3.5 \mathrm{TeV})$ can be probed at the $2 \sigma(5 \sigma)$ level, which is much beyond the LHC reach.

\section{ACKNOWLEDGMENTS}

We appreciate Manqi Ruan, Kepan Xie, and Fanrong $\mathrm{Xu}$ for useful discussions. This work was supported by the National Natural Science Foundation of China (NNSFC) under Grants No. 11947118, No. 12075300, No. 11821505, and No. 11851303, by Peng-Huan-Wu Theoretical Physics Innovation Center (No. 12047503), by the CAS Center for Excellence in Particle Physics (CCEPP), by the CAS Key Research Program of Frontier Sciences and from a Key R\&D Program of Ministry of Science and Technology under number 2017YFA0402204. C. H. acknowledges support from the Sun Yat-Sen University Science Foundation.
[1] G. Aad et al. (ATLAS Collaboration), J. High Energy Phys. 10 (2020) 062.

[2] G. Aad et al. (ATLAS Collaboration), Eur. Phys. J. C 80, 737 (2020).

[3] G. Aad et al. (ATLAS Collaboration), Phys. Rev. D 101, 072001 (2020).

[4] G. Aad et al. (ATLAS Collaboration), Phys. Rev. D 101, 052005 (2020).

[5] http://lepsusy.web.cern.ch/lepsusy.

[6] J. B. Guimarães da Costa et al. (CEPC Study Group), arXiv:1811.10545.

[7] T. Behnke et al., arXiv:1306.6327; H. Baer et al., arXiv: 1306.6352; C. Adolphsen et al., arXiv:1306.6353; C. Adolphsen et al., arXiv:1306.6328; T. Behnke et al., arXiv: 1306.6329.
[8] A. Abada et al. (FCC Collaboration), Eur. Phys. J. Special Topics 228, 261 (2019).

[9] M. Badziak, M. Olechowski, and P. Szczerbiak, Phys. Lett. B 770, 226 (2017).

[10] C. Han, Int. J. Mod. Phys. A 32, 1745003 (2017).

[11] M. Abdughani, L. Wu, and J. M. Yang, Eur. Phys. J. C 78, 4 (2018).

[12] H. Pagels and J. R. Primack, Phys. Rev. Lett. 48, 223 (1982).

[13] Y. Gu, M. Khlopov, L. Wu, J. M. Yang, and B. Zhu, Phys. Rev. D 102, 115005 (2020).

[14] J. E. Kim and H. P. Nilles, Phys. Lett. 138B, 150 (1984).

[15] G. Barenboim, E. J. Chun, S. Jung, and W. I. Park, Phys. Rev. D 90, 035020 (2014).

[16] M. Aaboud et al. (ATLAS Collaboration), Eur. Phys. J. C 76, 517 (2016). 
[17] P. Achard et al. (L3 Collaboration), Phys. Lett. B 587, 16 (2004).

[18] J. Alwall, M. Herquet, F. Maltoni, O. Mattelaer, and T. Stelzer, J. High Energy Phys. 06 (2011) 128.

[19] T. Sjöstrand et al., Comput. Phys. Commun. 191, 159 (2015).

[20] Y. Shen, H. Xiao, H. Li, S. Qin, Z. Wang, C. Wang, D. Zhang, and M. Ruan, Photon reconstruction performance at the CEPC baseline detector, arXiv:1908.09062.

[21] J. Gu and Y. Y. Li, Chin. Phys. C 42, 033102 (2018).

[22] M. Burns, K. Kong, K. T. Matchev, and M. Park, J. High Energy Phys. 03 (2009) 143.
[23] W. S. Cho, K. Choi, Y. G. Kim, and C. B. Park, Phys. Rev. Lett. 100, 171801 (2008).

[24] K. T. Matchev and M. Park, Phys. Rev. Lett. 107, 061801 (2011).

[25] P. Konar, K. Kong, K. T. Matchev, and M. Park, Phys. Rev. Lett. 105, 051802 (2010).

[26] G. Li, Y. n. Mao, C. Zhang, and S. h. Zhu, Phys. Rev. D 95, 035015 (2017).

[27] P. N. Bhattiprolu, S. P. Martin, and J. D. Wells, Eur. Phys. J. C 81, 123 (2021). 